\title{
Open-source dataset of vehicle state for an electric vehicle on a low-adhesion road
}

\author{
Shuo $\mathrm{CAI}^{1,2}$, Haitao DING ${ }^{*}$, Yunfeng $\mathrm{HU}^{1,2}$, Lin $\mathrm{ZHANG}^{3}$, Qin $\mathrm{LI}^{4,5}$ \& Hong CHEN ${ }^{2,3}$ \\ ${ }^{1}$ State Key Laboratory of Automotive Simulation and Control, Jilin University, Changchun 130025, China; \\ ${ }^{2}$ Department of Control Science and Engineering, Jilin University, Changchun 130025, China; \\ ${ }^{3}$ School of Automotive Studies, Tongji University, Shanghai 201804, China; \\ ${ }^{4}$ Collaborative Innovation Center of Electric Vehicles in Beijing, Beijing Institute of Technology, Beijing 100081, China; \\ ${ }^{5}$ GAC R\&D Center, Guangzhou 511434, China
}

Received 9 March 2020/Accepted 6 April 2020/Published online 29 January 2022

Citation Cai S, Ding H T, Hu Y F, et al. Open-source dataset of vehicle state for an electric vehicle on a low-adhesion road. Sci China Inf Sci, 2022, 65(3): 137201, https://doi.org/10.1007/s11432-020-3200-5

This study demonstrates and opens for academic research six sets of electric vehicle data collected during experiments on a low-adhesion road. The 11-dimensional data were collected from a small, single-motor SUV with front-wheel drive.

Experimental data collected for vehicles on low-adhesion roads are crucial for research on system dynamics modeling, stability control system design, and vehicle state estimation. Indeed, all vehicle control systems must be validated on low-adhesion roads, including vehicle models, control algorithms, and state estimation algorithms. It is very difficult to organize real vehicle experiments, considering experimental equipment, site selection, and cost. Therefore, it is impossible for most researchers to verify their algorithms using real vehicle experiments. One alternative solution is validation against existing data. Furthermore, in spite of the abundance of vehicle research, few scholars publish their original data, especially the data from low-adhesion roads.

The experimental data in this study are collected on compacted snow roads. Two kinds of experiments are included: double lane change and slalom. The data are stored in .mat format and can be directly read and processed in MATLAB software.

The 11 dimensions of experimental data are listed in Figure 1(a). $\delta_{s w}, \omega_{F L}, \omega_{F R}, \omega_{R L}, \omega_{R R}, a_{x}, a_{y}$ and $r$ are measured by the original sensors on the experimental vehicle. $\delta$ is calculated by $\delta_{s w}$ according to the steering ratio 15.65 , which is calculated from steering experimental data. $v_{x}$ and $v_{y}$ are measured by the additional high-precision combined inertial navigation system, RT3002, produced by Oxford Technical Solutions Ltd. The sampling resolution of the data is $0.01 \mathrm{~s}$. The basic parameters of the vehicle are listed in Figure 1(b).

The experimental data can be used in the development and verification of vehicle state estimation algorithms, such as tire-road friction coefficient, vehicle sideslip angle, and vehicle longitudinal and lateral velocities. Some noise in the measurement signals is difficult to describe with mathematical models, and the experimental data contains some nonlinear response of the vehicle. Therefore, these data can be used to verify the robustness of the estimation method of measuring noise and unmodeled dynamics; this verification is typically difficult to accomplish through the use of simulation data. By using these experimental data from a real vehicle, the effectiveness of the estimation method on the actual vehicle can be quickly verified, greatly shortening the development cycle for estimation methods. Moreover, the experimental data have the potential to be utilized for vehicle dynamics modeling and control system design and validation

Usage. In this study, a nonlinear observer is proposed to estimate the longitudinal and lateral velocities of the vehicle, and the estimation method is verified against experimental data.

The relationship between vehicle velocity, acceleration, and yaw rate is as follows:

$$
\dot{v}_{x}=a_{x}+r v_{y}, \quad \dot{v}_{y}=a_{y}-r v_{x} .
$$

If $a_{x}, a_{y}$, and $r$ are completely accurate, then $v_{x}$ and $v_{y}$ can be obtained by directly integrating according to (1). However, there is measurement noise in measurement signals $a_{x}$, $a_{y}$, and $r$, especially bias noise, causing the estimation results to diverge. Therefore, the following nonlinear observer is designed to solve this problem by applying corrections:

$$
\begin{gathered}
\dot{\hat{v}}_{x}=r \hat{v}_{y}+a_{x}+k_{x}\left(\frac{F_{x}\left(\hat{v}_{x}, \hat{v}_{y}\right)}{m}-a_{x}\right), \\
\dot{\hat{v}}_{y}=-r \hat{v}_{x}+a_{y}+k_{y}\left(\frac{F_{y}\left(\hat{v}_{x}, \hat{v}_{y}\right)}{m}-a_{y}\right),
\end{gathered}
$$

where $\hat{v}_{x}$ and $\hat{v}_{y}$ represent the respective estimated velocities, $F_{x}$ and $F_{y}$ are the longitudinal and lateral forces of the vehicle calculated from the vehicle model and the tire

*Corresponding author (email: dinght@jlu.edu.cn) 


\begin{tabular}{ccc}
\multicolumn{3}{c}{ Data list } \\
\hline Symbol & Description & Unit \\
\hline$\delta_{s w}$ & Steering wheel angle & $\circ$ \\
$\delta$ & Front wheel angle & $\circ$ \\
$\omega_{r L}$ & Angular velocity of front left wheel & $\mathrm{rad} / \mathrm{s}$ \\
$\omega_{r R}$ & Angular velocity of front right wheel & $\mathrm{rad} / \mathrm{s}$ \\
$\omega_{s L}$ & Angular velocity of rear left wheel & $\mathrm{rad} / \mathrm{s}$ \\
$\omega_{R R}$ & Angular velocity of rear right wheel & $\mathrm{rad} / \mathrm{s}$ \\
$a_{x}$ & Longitudinal acceleration & $\mathrm{m} / \mathrm{s}^{2}$ \\
$a_{y}$ & Lateral acceleration & $\mathrm{m} / \mathrm{s}^{2}$ \\
$r$ & Yaw angular velocity & $\mathrm{rad} / \mathrm{s}$ \\
$v_{x}$ & Longitudinal velocity & $\mathrm{m} / \mathrm{s}$ \\
$v_{y}$ & Lateral velocity & $\mathrm{m} / \mathrm{s}$ \\
\hline
\end{tabular}

(a)

\begin{tabular}{ccc}
\multicolumn{3}{c}{ Basic parameters of the vehicle } \\
\hline Parameter & Value & Unit \\
\hline Mass & 1669 & $\mathrm{~kg}$ \\
Moment of inertia & 2337.14 & $\mathrm{~kg} \cdot \mathrm{m}^{2}$ \\
Wheelbase & 2.56 & $\mathrm{~m}$ \\
Distance from center of mass to front axle & 1.153 & $\mathrm{~m}$ \\
Effective rolling radius of tire & 0.3231 & $\mathrm{~m}$ \\
Front track & 1.61 & $\mathrm{~m}$ \\
Rear track & 1.612 & $\mathrm{~m}$ \\
Height of center of mass & 0.601 & $\mathrm{~m}$ \\
Maximum road-tire friction coefficient & 0.4 & - \\
\hline
\end{tabular}

(b)

Dataset 1 I Dataset 2 I Dataset 3 I

:
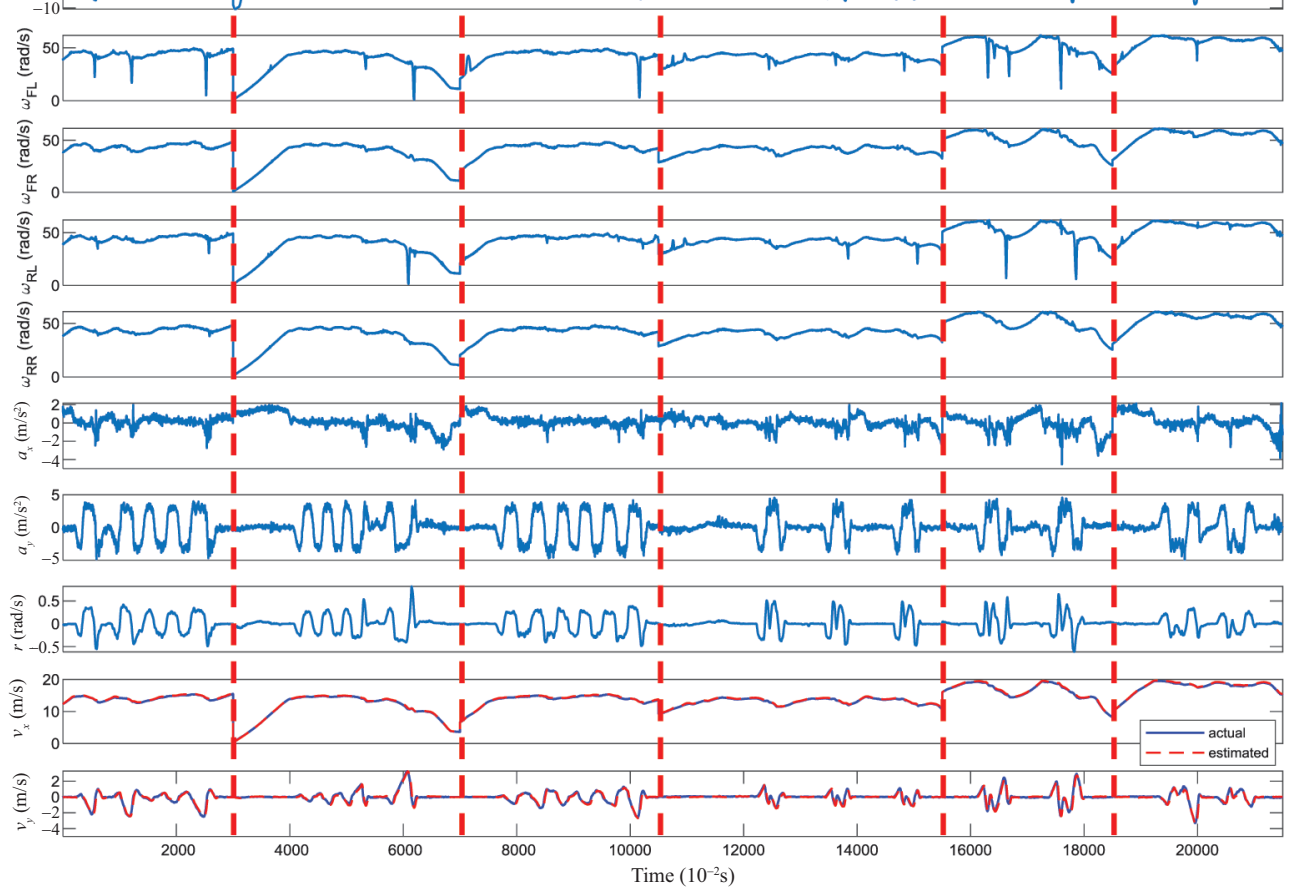

(c)

Figure 1 (Color online) (a) Data list; (b) basic parameters of the vehicle; (c) the experimental data and the estimated results.

model, $m$ is the mass of the vehicle, and $k_{x}$ and $k_{y}$ are the coefficients of the correction term. When selecting $k_{x}>0$ and $k_{y}>0$, the error dynamics system of this observer are input-to-state stable for the measurement signal noise and the vehicle model errors. For the experimental data in this study, $k_{x}=0.5, k_{y}=0.2$ are selected. The maximum estimation error of longitudinal velocity is $1.2 \mathrm{~m} / \mathrm{s}$, and the maximum estimation error of lateral velocity is $0.52 \mathrm{~m} / \mathrm{s}$.
The estimated effect is good, as shown in Figure 1(c).

Access methods. The experimental data can be download from the web ${ }^{1}$.

Acknowledgements This work was supported by National Natural Science Foundation of China (Grant Nos. U1864206, 61790564) and Shanghai Automotive Engineering Technology Development Foundation (Grant No. 1752). 